\title{
ADDENDUM TO A PAPER OF M. SAKS
}

\author{
by HAZEL PERFECT
}

(Received 20 June, 1982)

Throughout, $(X, \leq)$ denotes a partially ordered set (p.o. set), where $X$ is assumed to be finite. A subset $Y$ of $X$ is called a $k$-union if $Y$ contains no chain of length $k+1$. In particular, therefore, a 1-union is just an antichain; and it is readily seen that $Y$ is a $k$-union if and only if it is a union of $k$ antichains. (Dually, a subset $Z$ of $X$ is a $k$-counion if $Z$ contains no antichain of length $k+1$.) We denote by $d_{k}(X)$ the maximum cardinality of a $k$-union in $X$, with a similar notation for other p.o. sets. Now let $\mathscr{C}=\left\{C_{1}, \ldots, C_{q}\right\}_{\neq}$ be any partition of $X$ into chains, and write

$$
\beta_{k}(\mathscr{C})=\sum_{i=1}^{q} \min \left(k,\left|C_{i}\right|\right) .
$$

Since no $k$-union can intersect a chain in more than $k$ elements, it follows at once that

$$
d_{k}(X) \leq \beta_{k}(\mathscr{C})
$$

A chain partition $\mathscr{C}$ for which

$$
d_{k}(X)=\beta_{k}(\mathscr{C})
$$

is called $k$-saturated. In [3], Greene and Kleitman have shown that $k$-saturated partitions exist for every positive integer $k$ and, in [4], Saks has provided a much simpler and very elegant proof of this basic result. The case $k=1$ is, of course, Dilworth's theorem [1]. In fact, Greene and Kleitman proved a stronger theorem, namely that there always exists a chain partition of $X$ which is simultaneously $k$ - and $(k+1)$-saturated. It would appear that the theory of counions depends on this stronger theorem, and it is our purpose in this short note to point out how the method of Saks can easily be exploited to obtain this generalization.

Following Saks, let us denote by $[k]$ the p.o. set on $\{1,2, \ldots, k\}$ with the natural ordering, and let $X \times[k]$ be the usual product set, i.e. it consists of all pairs $(x, i)$ with $x \in X$ and $1 \leq i \leq k$, and is made into a p.o. set with respect to componentwise order. In this latter p.o. set, we shall say that an element $(x, i)$ is at level $i$.

LEMMA 1. An antichain of length $p\left(>d_{1}(X \times[k])\right)$ in the p.o. set $X \times[k+1]$ contains at least $p-d_{1}(X \times[k])$ elements at level 1 .

For, let $A$ be such an antichain containing just $n$ elements at level 1 , and let the set of these elements be $A^{\prime}$. Then $A \backslash A^{\prime}$ is an antichain in $X \times[k+1] \backslash X \times[1]$. But this p.o. set is isomorphic to $X \times[k]$, and so

$$
\left|A \backslash A^{\prime}\right| \leq d_{1}(X \times[k]),
$$

Glasgow Math. J. 25 (1984) 31-33. 
from which we deduce that

$$
n \geq p-d_{1}(X \times[k]) .
$$

It is Lemma 2 below which is the crux of the argument. Before stating it we recall a result on maximum-sized antichains. Let the set of all these in a given p.o. set be $\mathscr{Y}$. A relation $\leqslant$ defined on $\mathscr{S}$ by the rule

$$
A \leqslant A^{\prime} \Leftrightarrow \text { for each } a \in A \text {, there exists } a^{\prime} \in A^{\prime} \text { with } a \leq a^{\prime}
$$

is itself a p.o. on $\mathscr{S}$, and with respect to $\leqslant$ there exists a (unique) greatest member of $\mathscr{S}$, i.e. a 'top' antichain $A^{+} \in \mathscr{S}$ such that $A \leqslant A^{+}$for every $A \in \mathscr{Y}$. (See, for example, [2].) In the statement of the lemma, we write $m_{k}=d_{1}(X \times[k+1])-d_{1}(X \times[k])$. Also, a chain partition which contains as few chains as possible is called a minimum chain partition.

LEMMA 2. There exists a minimum chain partition of $X \times[k+1]$ which contains at least (and so exactly) $m_{k}$ chains consisting entirely of elements at level 1 .

Let $\mathscr{C}$ be a minimum chain partition of $X \times[k+1]$ which contains just $s$ chains consisting entirely of elements at level 1 . If $s<m_{k}$, we shall show how to increase this number by 1 . Write $\mathscr{C}=\left\{C_{1}, \ldots, C_{s}, C_{s+1}, \ldots, C_{q}\right\}_{\neq}$, where $q=d_{1}(X \times[k+1])$ and where $C_{1}, \ldots, C_{s}$ consist entirely of elements at level 1 , and let $X^{\prime}=X \times[k+1] \backslash\left(C_{1} \cup \ldots \cup C_{s}\right)$. Now $X^{\prime}$ is covered by $q-s$ chains and by no fewer; so any maximum antichain in $X^{\prime}$ has length $q-s$ (by Dilworth's theorem). Let $A^{+}$be the top maximum antichain in $X^{\prime}$. By Lemma $1, A^{+}$contains at least $(q-s)-d_{1}(X \times[k])=m_{k}-s(>0)$ elements at level 1 . Suppose $a$ is one of these and assume $a \in C_{s+1}$. Write $C_{s+1}=C_{s+1}^{-} \cup C_{s+1}^{+}$, where $C_{s+1}^{-}$ consists of all elements of $C_{s+1}$ below and including $a$, and write $X^{\prime \prime}=X^{\prime} \backslash C_{s+1}^{-}$. Now in $X^{\prime \prime}$ there can be no antichain of length $q-s$. For if $A$ were such a one then $A$ must contain an element $b$ (say) from $C_{s+1}^{+}$, and, since $A \leqslant A^{+}$, there exists $c \in A^{+}$with $b \leq c$. Since $b>a$, we have $a<c, a, c \in A^{+}$, which is a contradiction. Therefore $X^{\prime \prime}$ can be covered by $q-s-1$ chains $C_{s+2}^{\prime}, \ldots, C_{q}^{\prime}$ (say), and finally $\left\{C_{1}, \ldots, C_{s}, C_{s+1}^{-}, C_{s+2}^{\prime}, \ldots, C_{q}^{\prime}\right\}$ is a minimum chain partition of $X \times[k+1]$ with the first $s+1$ chains consisting entirely of elements at level 1 .

The proof of the general theorem of Greene and Kleitman now follows from the sequence of immediately verifiable statements I-V below. We use the term 'special' for those chain partitions of $X \times[k+1]$ which Saks called 'associable'.

I. There exists a minimum special chain partition of $X \times[k+1]$ in which $m_{k}$ chains consist only of elements at level 1 .

If $\mathscr{C}=\left\{C_{1}, \ldots, C_{m_{k}}, C_{m_{k}+1}, \ldots, C_{q}\right\}_{\neq}$is such a partition, where $C_{1}, \ldots, C_{m_{k}}$ consist entirely of elements at level 1 , then $\mathscr{C}^{\prime}=\left\{C_{1}^{\prime}, \ldots, C_{q}^{\prime}\right\}$, where $C_{i}^{\prime}=\left\{(x, j):(x, j+1) \in C_{i}\right\}$ $(1 \leq i \leq q)$, is such that $C_{1}^{\prime}=\ldots=C_{m_{k}}^{\prime}=\varnothing$. Denote by $\mathscr{C}^{\prime \prime}$ the collection of non-empty members of ' $\mathscr{b}$.

II. $\mathscr{C}^{\prime \prime}$ is a chain partition of $X \times[k]$.

III. $\mathscr{C}^{\prime \prime}$ is minimum.

IV. $\mathscr{C}^{\prime \prime}$ is special. 
$V$. If $\mathscr{C}^{*}$ is the chain partition of $X$ which is associated (in the sense in which Saks uses this term) with $\mathscr{C}$, then $\mathscr{C}^{*}$ is also associated with $\mathscr{C}^{\prime \prime}$, and therefore

$$
\beta_{k}\left(\mathscr{C}^{*}\right)=d_{k}(X) \text { and } \beta_{k+1}\left(\mathscr{C}^{*}\right)=d_{k+1}(X) \text {. }
$$

\section{REFERENCES}

1. R. P. Dilworth, A decomposition theorem for partially ordered sets, Ann. of Math. 51 (1950), 161-166.

2. R. Freese, An application of Dilworth's lattice of maximal antichains, Discrete Math. 7 (1974), 107-109.

3. C. Greene and D. J. Kleitman, The structure of Sperner $k$-families, J. Combin. Theory Ser. A 20 (1976), 41-68.

4. M. Saks, A short proof of the existence of $k$-saturated partitions of partially ordered sets, Adv. in Math. 33 (1979), 207-211.

UNIVERSITY OF SHEFFIELD 